\title{
Exact calculation of Fourier series in nonconforming spectral-element methods
}

\author{
Aimé Fournier \\ NCAR Institute for Mathematics Applied to Geosciences \\ Boulder CO 8030\%-3000 USA
}

\begin{abstract}
Key words: adaptive mesh refinement, Fourier analysis, spectral-element method PACS: 02.30.Nw, 02.60.Cb, 02.60.Jh, 02.70.Dh, 02.70.Jn
\end{abstract}

\section{Usefulness of calculating Fourier series in the SEM}

In this note is presented a method, given nodal values on multidimensional nonconforming spectral elements, for calculating global Fourier-series coefficients. This method is "exact" in that given the approximation inherent in the spectral-element method (SEM), no further approximation is introduced that exceeds computer round-off error. The method is very useful when the SEM has yielded an adaptive-mesh representation of a spatial function whose global Fourier spectrum must be examined, e.g., in dynamically adaptive fluiddynamics simulations such as [7].

Email address: fournier@ucar .edu (Aimé Fournier).

URL: http://www. asp.ucar.edu/gtp/fournier (Aimé Fournier).

Preprint submitted to J. Comp. Phys.

2005 June 2 


\section{Derivation of an exact transform}

Suppose we have some functional problem in a spatial domain $\mathbb{D}:=[-\pi, \pi]^{d}$ (possibly including toroidal geometry) and use coordinate transformations

$$
\vec{\vartheta}_{k} \quad \text { from } \quad \vec{\xi} \in \mathbb{E}_{0}:=[-1,1]^{d} \quad \text { to } \quad \vec{x} \in \mathbb{E}_{k}
$$

to partition $\mathbb{D}=\bigcup_{k=1}^{K} \mathbb{E}_{k}$ by $K$ elements $\mathbb{E}_{k}:=\vec{\vartheta}_{k}\left(\mathbb{E}_{0}\right)$ with disjoint ${ }^{1}$ interiors. Typically the SEM approximates the exact solution by its piecewise polynomial representation of degree $P$ :

$$
u^{\mathrm{ex}}(\vec{x}) \approx u(\vec{x})=\sum_{k=1}^{K} \sum_{\vec{\jmath} \in \mathbb{J}} u_{\vec{\jmath}, k} \phi_{\vec{\jmath}, k}(\vec{x}),
$$

where $\mathbb{J}:=\{0, \ldots P\}^{d}$ indexes the values $u_{\vec{\jmath}, k}:=u\left(\vec{x}_{\vec{\jmath}, k}\right)$ and nodes $\vec{x}_{\vec{\jmath}, k}:=\vec{\vartheta}_{k}\left(\vec{\xi}_{\vec{\jmath}}\right)$ mapped from the $d$-dimensional Gauss-Lobatto-Legendre (GLL) quadrature $\operatorname{nodes} \xi_{\vec{\jmath}}^{\alpha}:=\xi_{\jmath^{\alpha}} \in[-1,1]$,

$$
\phi_{\vec{\jmath}, k}(\vec{x}):= \begin{cases}\phi_{\vec{\jmath}} \circ \vec{\vartheta}_{k}^{-1}(\vec{x}), & \vec{x} \in \mathbb{E}_{k} \\ 0, & \vec{x} \notin \mathbb{E}_{k}\end{cases}
$$

is the $\vec{x}_{\vec{\jmath}, k}$-interpolating piecewise-polynomial,

$$
\phi_{\vec{\jmath}}(\vec{\xi}):=\prod_{\alpha=1}^{d} \phi_{j^{\alpha}}\left(\xi^{\alpha}\right) \quad \text { and } \quad \phi_{j}(\xi):=\sum_{p=0}^{P} \check{\phi}_{j, p} \mathrm{~L}_{p}(\xi)
$$

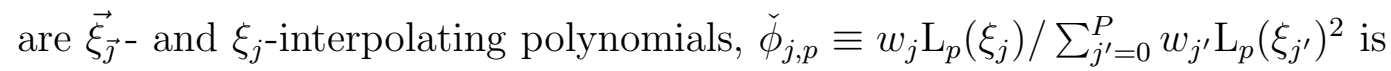
a Legendre coefficient [e.g., $4,(B .3 .15)], \sqrt{p+\frac{1}{2}} \mathrm{~L}_{p}(\xi)$ is the orthonormal Legendre polynomial of degree $p$ on $[-1,1]$ and $w_{j}$ is the GLL quadrature weight. In many cases a physically interesting quantity is the global Fourier-series coefficient $\hat{u}_{\vec{q}}$ at integer wavenumber components $q^{\alpha}$, usually approximated by

$\overline{1 \dot{\mathbb{E}}_{k} \cap \dot{\mathbb{E}}_{k^{\prime}}=\varnothing \text { if } k \neq k^{\prime}}$ 
$M^{d}$-point trigonometric $d$-cubature in such manner as

$$
\begin{aligned}
\hat{u}_{\vec{q}} & :=\frac{1}{(2 \pi)^{d}} \int_{\mathbb{D}} u(\vec{x}) \mathrm{e}^{-\mathrm{i} \vec{q} \cdot \vec{x}} \mathrm{~d} v(\vec{x}) \equiv \sum_{k=1}^{K} \sum_{\vec{\jmath} \in \mathbb{J}} \hat{\phi}_{\vec{\jmath}, k, \vec{q}} u_{\vec{\jmath}, k}, \\
\text { where } \quad \hat{\phi}_{\vec{\jmath}, k, \vec{q}} & =\frac{1}{M^{d}} \sum_{\vec{m} \in \mathbb{M}} \phi_{\vec{\jmath}, k}\left(\vec{x}_{\vec{m}}\right) \mathrm{e}^{-\mathrm{i} \vec{q} \cdot \vec{x}_{\vec{m}}}-\mathcal{E}_{\vec{q}} \phi_{\vec{\jmath}, k},
\end{aligned}
$$

$\mathrm{d} v(\vec{x}):=\prod_{\alpha=1}^{d} \mathrm{~d} x^{\alpha}$ is the volume differential and $\mathbb{M}:=\{1, \ldots M\}^{d}$ indexes trigonometric nodes $x_{\vec{m}}^{\alpha}:=\left(2 m^{\alpha} / M-1\right) \pi$. Note whenever $\mathbb{D}$ is adaptively repartitioned there is an additional computation cost of $\mathcal{O}\left(M^{d}\right)$ per node to use (2) to provide in (6) the values $\phi_{\vec{\jmath}, k}\left(\vec{x}_{\vec{m}}\right)$, as well as a $d$-cubature error [generalizing 3, theorem 4.7]

$$
\mathcal{E}_{\vec{q}} u \equiv \sum_{\vec{r} \in \mathbb{Z}^{d} \backslash\{\overrightarrow{0}\}} \hat{u}_{\vec{q}+M \vec{r}}
$$

that in general converges no faster than $\mathcal{O}\left(M^{-2}\right)$, because $\mathbb{C}^{1}$ discontinuities of (2) across element boundaries cause $\left|\hat{u}_{\vec{q}}\right|$ to decay only as $\mathcal{O}\left(|\vec{q}|^{-2}\right)$. We discover a more accurate method by substituting (3) into (5) to yield

$$
\begin{aligned}
\hat{\phi}_{\vec{\jmath}, k, \vec{q}}= & \frac{1}{(2 \pi)^{d}} \int_{\mathbb{E}_{k}} \mathrm{e}^{-\mathrm{i} \vec{q} \cdot \vec{x}} \phi_{\vec{\jmath}} \circ \vec{\vartheta}_{k}^{-1}(\vec{x}) \mathrm{d} v(\vec{x}) \\
& \stackrel{(1)}{=} \frac{1}{(2 \pi)^{d}} \int_{\mathbb{E}_{0}} \mathrm{e}^{-\mathrm{i} \vec{q} \cdot \vec{\vartheta}_{k}(\vec{\xi})} \phi_{\vec{\jmath}}(\vec{\xi})\left|\frac{\partial \vec{\vartheta}_{k}}{\partial \vec{\xi}}\right| \mathrm{d} v(\vec{\xi}) \\
& \stackrel{(4)}{=} \frac{1}{(2 \pi)^{d}} \int_{\mathbb{E}_{0}} \mathrm{e}^{-\mathrm{i} \cdot \vec{\vartheta}_{k}(\vec{\xi})}\left(\prod_{\alpha=1}^{d} \sum_{p=0}^{P} \check{\phi}_{\jmath^{\alpha}, p} \mathrm{~L}_{p}\left(\xi^{\alpha}\right)\right)\left|\frac{\partial \vec{\vartheta}_{k}}{\partial \vec{\xi}}\right| \mathrm{d} v(\vec{\xi}) .
\end{aligned}
$$

In many applications, especially when $u$-structure rather than domain geometry is guiding the mesh adaption, each $\mathbb{E}_{k}$ is a $d$-parallelepiped with center $\vec{a}_{k}$ and $d$ legs $2 \vec{h}_{k}^{\alpha}$, so we have an affinity $\vec{\vartheta}_{k}(\vec{\xi}):=\vec{a}_{k}+\overrightarrow{\vec{h}}_{k} \cdot \vec{\xi}$, where $\vec{h}_{k}^{\alpha}$ make up the columns of $\overrightarrow{\vec{h}}_{k}$. Then we obtain

$$
\hat{\phi}_{\vec{\jmath}, k, \vec{q}}=\frac{1}{(2 \pi)^{d}}\left|\overrightarrow{\vec{h}}_{k}\right| \mathrm{e}^{-\mathrm{i} \vec{q} \cdot \vec{a}_{k}} \prod_{\alpha=1}^{d} \sum_{p=0}^{P} \check{\phi}_{\jmath^{\alpha}, p} \int_{-1}^{1} \mathrm{e}^{-\mathrm{i} \vec{q} \cdot \vec{h}_{k}^{\alpha} \xi} \mathrm{L}_{p}(\xi) \mathrm{d} \xi
$$


Finally, recalling the classical identity [e.g., 1, exercise 12.4.9] for the spherical Bessel function $\mathrm{B}_{p}(r)$ of the first kind,

$$
\begin{gathered}
\mathrm{B}_{p}(r) \equiv \frac{\mathrm{i}^{p}}{2} \int_{-1}^{1} \mathrm{e}^{-\mathrm{i} r \xi} \mathrm{L}_{p}(\xi) \mathrm{d} \xi \\
\text { we obtain } \quad \hat{\phi}_{\vec{\jmath}, k, \vec{q}}=\frac{1}{\pi^{d}}\left|\overrightarrow{\vec{h}}_{k}\right| \mathrm{e}^{-\mathrm{i} \vec{q} \cdot \vec{a}_{k}} \prod_{\alpha=1}^{d} \sum_{p=0}^{P} \check{\phi}_{\jmath^{\alpha}, p} \mathrm{i}^{-p} \mathrm{~B}_{p}\left(\vec{q} \cdot \vec{h}_{k}^{\alpha}\right) .
\end{gathered}
$$

Note that most expressions in (8) can be precomputed; objects that may vary during a dynamically adaptive computation, such as $\vec{a}_{k}$ or $\vec{h}_{k}^{\alpha}$, typically take values from a sparse set, e.g., a collection of powers of 2 . The computation of (5) now incurs no additional error beyond that of (2). Also note, to generalize to the case $P=P_{k}^{\alpha}$ is straightforward.

\section{Accuracy of transform for 1D \& 2D test cases}

Equation (8) was implemented in MatLab ${ }^{\circledR}$ and tested using known results for (5). The most immediate test follows from (7), namely $\left.\hat{u}_{q}^{\mathrm{ex}}=\widehat{\mathrm{L}_{\mathrm{p}}(\cdot / \pi}\right)_{q}=$ $\mathrm{i}^{-p} \mathrm{~B}_{p}(\pi q)$. In this case (5) was found to reproduce (7) to 12-16 digits for $K=1, P \leq 18$, implying similar performance for any polynomial $u(\vec{x})$ in this range. The next test was to put $u^{\mathrm{ex}}(x)=\sin x$, or $\hat{u}_{q}^{\mathrm{ex}}=\left(\delta_{q, 1}-\delta_{q,-1}\right) / 2 \mathrm{i}$. Since this is not a polynomial we should expect at best to see algebraic convergence w.r.t. $K$ in a uniform meshing $a_{k}=(k-1) h_{k}-\pi, h_{k}=2 \pi / K$ and exponential convergence w.r.t. $P$, as verified in Fig. 1 . Note there is no need to test $u^{\mathrm{ex}}(x)=$ $\sin r x$ for $r>1$ because of scaling.

We conclude by examining three $2 \mathrm{D}$ tests with adaptive meshing in the fashion of [5], using MatLab ${ }^{\circledR}$. Fig. 2 confirms (5) in the case [6, (19)]

$$
u^{\mathrm{ex}}(\vec{x}) \equiv \sum_{\vec{q} \in \mathbb{Z}^{2}} \mathrm{e}^{b^{1}\left|q^{1}\right|+b^{2}\left|q^{2}\right|+\mathrm{i} \vec{q} \cdot \vec{l} \cdot \vec{x}}
$$




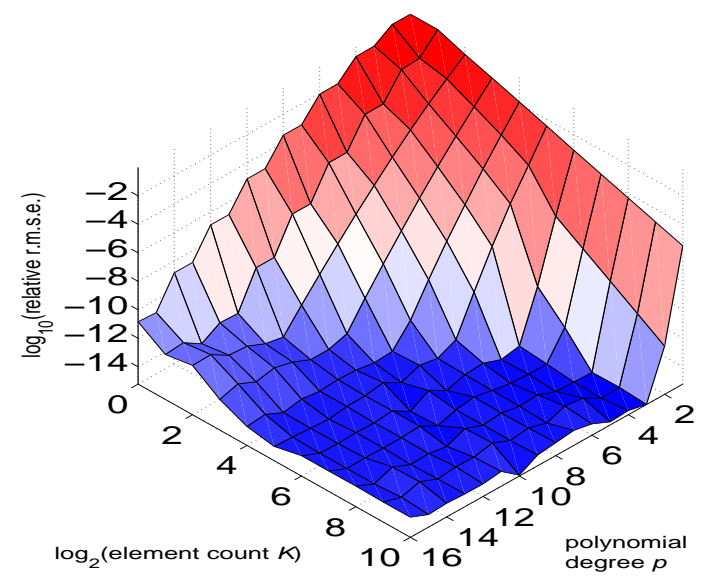

Fig. 1. Surface plot (blue low to red high) of $\log _{10}$ relative r.m.s. error in (5) for $u^{\mathrm{ex}}(x)=\sin x, \operatorname{vs} \log _{2} K$ and $P$.

where $b^{\alpha}=-\frac{2}{5}$ and $\overrightarrow{\vec{l}} \doteq\left(\begin{array}{rr}l^{1} & l^{2} \\ -l^{2} & l^{1}\end{array}\right)=\left(\begin{array}{rr}1 & 2 \\ -2 & 1\end{array}\right)$ is a biperiodicity-preserving "rotation". As expected, the red curve (connecting the $\left|\hat{u}_{\vec{q}}\right|$ peaks) shows a powerlaw decay in $\vec{q}$-space. Note, in this plot and those below the $\overrightarrow{\vec{l}}$-operation helps instigate mesh adaption but has the consequence of leaving $\vec{q}$ undersampled in $\mathbb{Z}^{2}$. In Fig. 3 is shown an initial condition $[6,(22)]$

$$
\vec{u}^{\mathrm{ex}}(0, \vec{x}):=-\vec{l} \sin \vec{l} \cdot \vec{x}
$$

for the 2D Burgers eq. As expected, $\hat{u}_{\vec{q}}$ almost vanishes for $\vec{q} \neq \pm \vec{l}$. Finally, at time $t=1.6037 / \pi|\vec{l}|^{2}$ the analytic solution generalizing $[2,(2.5)]$ to $2 \mathrm{D}$ is shown in Fig. 4. As expected for the nearly $\mathbb{C}^{0}$-discontinuous fronts $\perp \vec{l}$ seen at left, $\left|\hat{u}_{\vec{q}}^{1}\right|$ decays slightly faster than $\mathcal{O}\left(|\vec{q}|^{-1}\right)$ but only for wavevectors $\vec{q} \| \vec{l}$ (red curve).

\section{References}

[1] George Arfken, 1985: Mathematical methods for physicists, 3rd ed., Academic Press, 985 pp. 


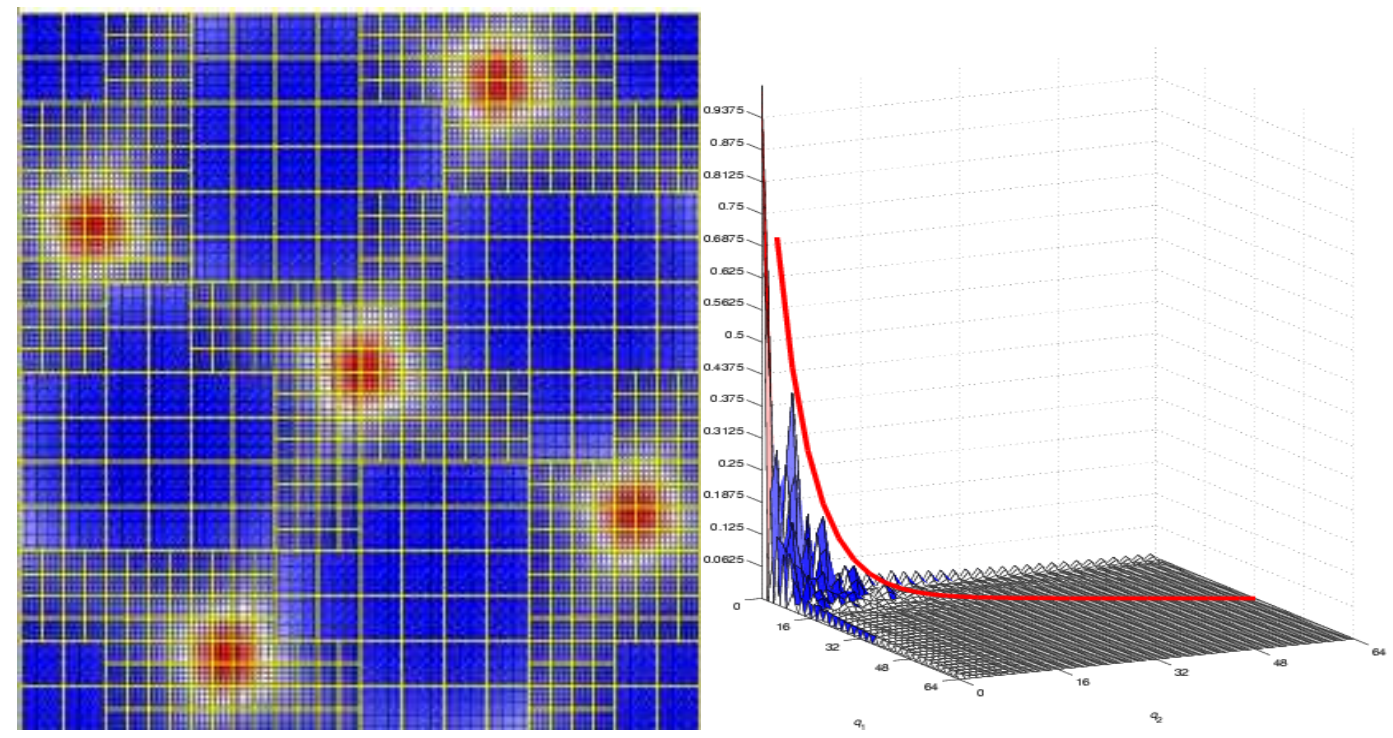

Fig. 2. Left, $u(9)$ over the spatial $\vec{x}$ domain, increasing from blue to red; yellow lines indicate element boundaries, black lines show nodes $\vec{x}_{\vec{\jmath}, k}$ with $P=5$. Right, surface plot of $\left|\hat{u}_{\vec{q}}\right|$ from (5) vs $q^{1}$ and $q^{2}$.

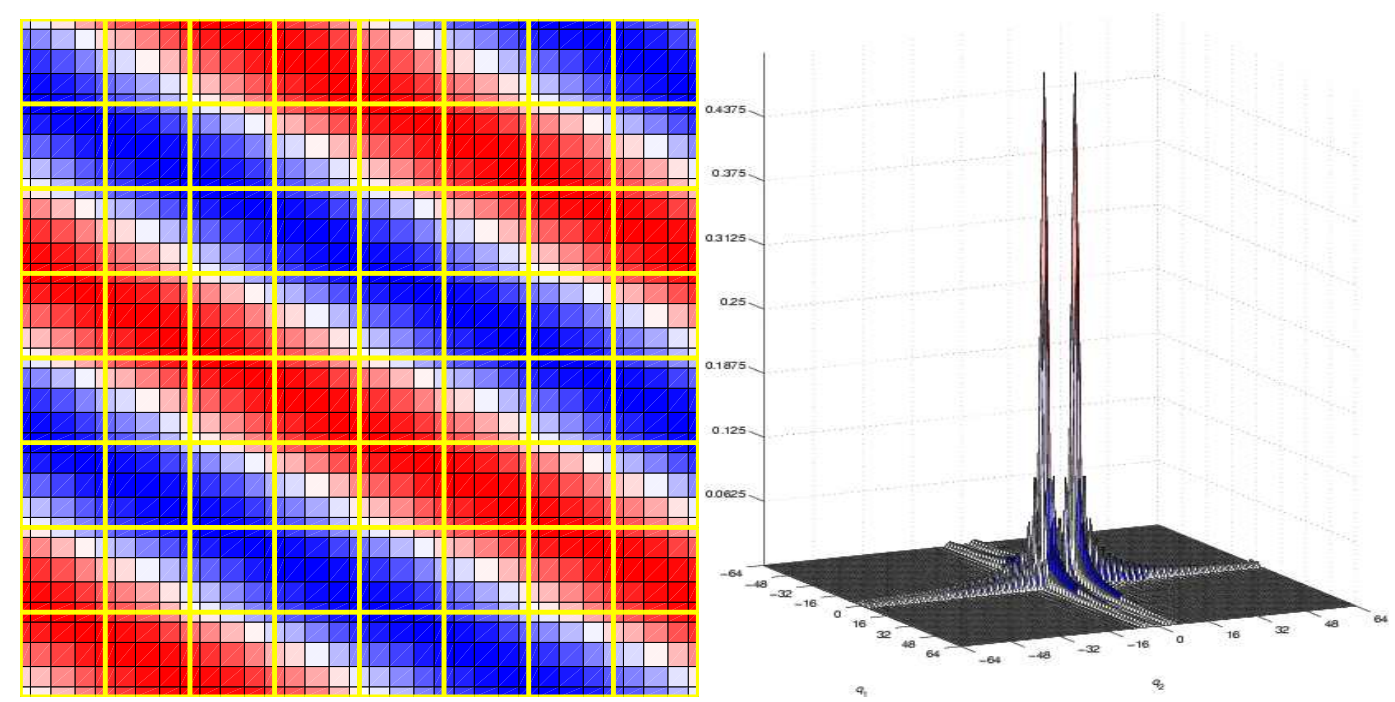

Fig. 3. As in Fig. 2 but for the $t=0$ state given by (10), in $K=2^{6}$ elements.

[2] C. Basdevant, M. Deville, P. Haldenwang, J.M. Lacroix, J. Ouazzani, R.

Peyret \& P. Orlandi, 1986: Spectral and finite difference solutions of the

Burgers equation. Computers $\&$ Fluids, 14, 23-41.

[3] John P. Boyd, 1989: Chebyshev \& Fourier spectral methods, Springer, 793

pp. 


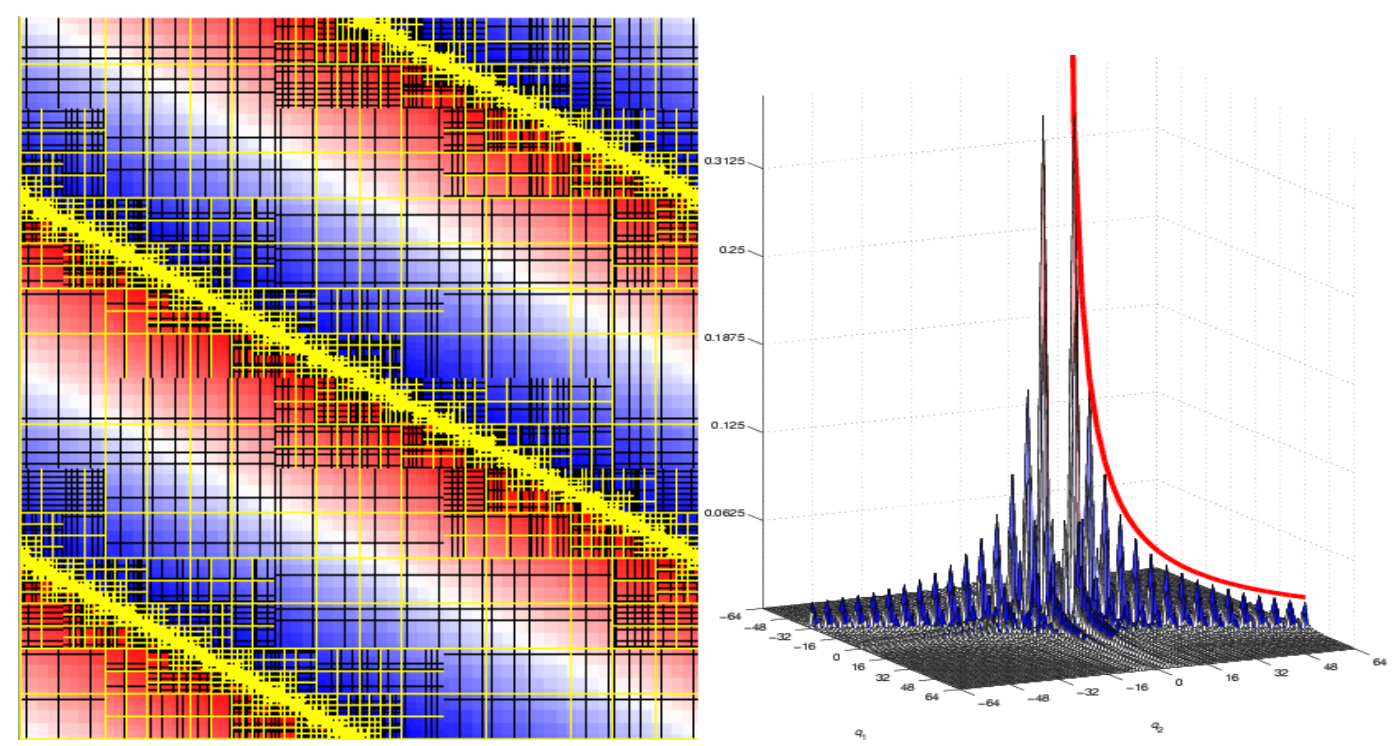

Fig. 4. As in Fig. 3 but for $t=1.6037 / 5 \pi$.

[4] M.O. Deville, P.F. Fischer \& E.H. Mund, 2002: High-order methods for incompressible fluid flow, Cambridge, $499 \mathrm{pp}$.

[5] A. Fournier and D. Rosenberg, 2005: "Multiresolution adaptive spectralelement modeling of geophysical fluid dynamics using GASpAR," in preparation.

[6] Fournier, A., G. Beylkin and V. Cheruvu, 2005: Multiresolution adaptive space refinement in geophysical fluid dynamics simulation. Lecture Notes Comp. Sci. Eng., 41, 161-170.

[7] Rosenberg, D., A. Fournier, P.F. Fischer \& A. Pouquet, 2005: Geophysicalastrophysical spectral-element adaptive refinement (GASpAR): Objectoriented $h$-adaptive code for geophysical fluid dynamics simulation. $J$. Comp. Phys., submitted. 